\title{
Editorial: Inter-Organelle Calcium Communication in Cancer
}

\author{
Cesar Cardenas ${ }^{1,2,3,4 *}$, Paolo Pinton ${ }^{5,6}$ and Geert Bultynck $k^{7,8}$ \\ ${ }^{1}$ Anatomy and Developmental Biology Program, Institute of Biomedical Sciences, University of Chile, Santiago, Chile, \\ ${ }^{2}$ Geroscience Center for Brain Health and Metabolism, Santiago, Chile, ${ }^{3}$ Buck Institute for Research on Aging, Novato, \\ CA, United States, ${ }^{4}$ Department of Chemistry and Biochemistry, University of California, Santa Barbara, Santa Barbara, CA, \\ United States, ${ }^{5}$ Department of Morphology, Surgery and Experimental Medicine, Section of Pathology, Oncology and \\ Experimental Biology, Laboratory for Technologies of Advanced Therapies (LTTA), University of Ferrara, Ferrara, Italy, \\ ${ }^{6}$ Maria Cecilia Hospital, GVM Care \& Research, E.S: Health Science Foundation, Cotignola, Italy, ${ }^{7}$ Laboratory of Molecular \\ and Cellular Signaling, Department Cellular and Molecular Medicine, KU Leuven, Leuven, Belgium, ${ }^{8}$ Leuven Kanker Instituut \\ (LKI), KU Leuven, Leuven, Belgium
}

Keywords: mitochondria, endoplasmic reticulum, MAMs, IP3R, mitochondrial Ca2+ uniporter, BCL-2, metastasis, invasion

Editorial on the Research Topic

Inter-Organelle Calcium Communication in Cancer

\section{INTRODUCTION}

Cancer is a leading cause of death worldwide, accounting for 8.2 million deaths (around 13\% of all deaths) in 2012, with this number expected to rise to over 11 million by 2030 (1). The term "cancer" groups over 100 distinct diseases that share a series of "acquired capabilities," which were first defined by Hanahan and Weinberg in 2000 consisting of six hallmark alterations in cell physiology that collectively dictate malignant growth: cell sufficiency in growth signals, insensitivity to growth inhibitory signals, evasion of programmed cell death, limitless replicative potential, sustained angiogenesis, and tissue invasion and metastasis (2). In the last 25 years, the description and interpretation of genomic abnormalities in cancer cells have dominated cancer research (3). However, bioinformatic analyses suggest that cancer-related driver mutations affect a dozen or more core cell signaling pathways (4), suggesting that individual targets will not be fruitful candidates for therapeutic development, and instead, intervening with cellular processes will be more effective. In this regard, it has become increasingly clear that many key oncogenic signaling pathways that support tumor cell growth and survival converge at cell metabolism (5). In fact, the reprogramming of energy metabolism, which relies on mitochondrial function, is absolutely required for malignant transformation, and for this reason, it has recently been added as a hallmark of cancer (6). This research topic focuses on the role of the endoplasmic reticulum (ER)-to-mitochondria communication in the regulation of mitochondrial function, with special emphasis on $\mathrm{Ca}^{2+}$ transfer, and its impact on cancer cell fate.

\section{MITOCHONDRIA-ASSOCIATED ER MEMBRANES IN CANCER}

Received: 11 January 2018 Accepted: 17 January 2018 Published: 06 February 2018

Citation:

Cardenas C, Pinton P and Bultynck G (2018) Editorial: Inter-Organelle Calcium Communication in Cancer. Front. Oncol. 8:14. doi: 10.3389/fonc.2018.00014
It has been known since the 1950 that mitochondria and the ER, the main intracellular $\mathrm{Ca}^{2+}$ store, interact with each other in response to changes in cellular metabolism (7). The ER-mitochondrial association can be physically isolated (8) as mitochondria-associated ER membranes that establish a specific microdomain with distinct signaling functions (9). These contact sites not only allow "quasi-synaptic" transfer of $\mathrm{Ca}^{2+}$ from the ER to the mitochondria but also enables lipid synthesis and exchange, mitochondrial trafficking, cell death, bioenergetics, proteostasis, and autophagy, all processes often altered in oncogenesis and cancer, as has been reviewed in detail by Sassano et al. 
Consistent with the pivotal role of MAMs in these fundamental cellular processes, their function and thus composition ought to be fine-tuned. This is achieved by a growing list of proteins that impact cell survival and cell stress tolerance, of which, many are well-known oncogenes and oncosuppressors such as p53, AKT, PTEN, and PML $(10,11)$. Pedriali et al. discuss how these proteins modulate $\mathrm{Ca}^{2+}$ homeostasis. In addition, they present an exhaustive summary of drugs that target $\mathrm{Ca}^{2+}$ transport being used as anticancer therapies. Also, several Bcl-2-family members, critical regulators of cell death and often dysregulated in cancer, have been identified at the MAMs, impacting cell survival through the regulation of ER-mitochondrial $\mathrm{Ca}^{2+}$ dynamics, a topic further contemplated by Vervliet et al. Herrera-Cruz and Simmen go a step further and consider the role of structural proteins that enable the formation of MAMs; e.g., mitofusin-2, phosphofurin acidic cluster sorting protein 2 (PCS-2), and Nogo-B/reticulon$4 \mathrm{~B}$ as oncogenes or oncosuppressors. As mentioned, MAMs actively participate in the regulation of proteostasis, tuning the ER stress response to cope with metabolic demand under various stress stimuli. Carreras-Sureda et al. immerses us in the unfold protein response, its relation with MAMs, and the role this plays in cancer.

\section{$\mathrm{Ca}^{2+}$ BASIC TOOLKIT; IP3R, VDAC, AND MITOCHONDRIAL $\mathrm{Ca}^{2+}$ UNIPORTER (MCU) IN CANCER}

$\mathrm{Ca}^{2+}$ is a ubiquitous signaling messenger that regulates various cellular processes ranging from muscle contraction to synaptic transmission and from cellular proliferation to cell death $(12,13)$. This versatility is achieved by the interplay of the $\mathrm{Ca}^{2+}$-signaling toolkit (including channels, pumps, exchangers, and binding/ buffering proteins), generating specific spatiotemporal $\mathrm{Ca}^{2+}$ signals (14). Alterations in the expression and/or function of these $\mathrm{Ca}^{2+}$-transport/binding systems have been implicated in oncogenesis and cancer progression $(15,16)$. In this regard, Wolf and Guse contribute a comprehensive review focused on the generation, regulation, and function of $\mathrm{Ca}^{2+}$ microdomains in $\mathrm{T}$ lymphocytes. $\mathrm{Ca}^{2+}$ microdomains occur in cancer cells due to the spontaneous opening of a single or some small clusters of the inositol 1,4,5-trisphosphate (IP3) receptor (IP3R), in the $\mathrm{ER}$, which proves to be necessary to maintain the bioenergetic homeostasis of these cells (17), as deliberated by Bustos et al. $\mathrm{Ca}^{2+}$ regulates bioenergetics and metabolism by entering the mitochondria where it modulates the activity of key enzymes of the tricarboxylic acid cycle and fatty acid oxidation (18). $\mathrm{Ca}^{2+}$ crosses the mitochondrial outer membrane through the voltagedependent anion channel 1 (VDAC1), a highly $\mathrm{Ca}^{2+}$ permeable channel that controls metabolism and bioenergetics by modulating the access of $\mathrm{Ca}^{2+}$ as well as pyruvate, malate, succinate, and NADH to the inner mitochondrial membrane. ShoshanBarmatz et al. review the role of VDAC1 in cancer with special emphasis on its role in apoptosis induced by $\left[\mathrm{Ca}^{2+}\right]$-increasing agents, which upregulate VDAC1 expression and subsequently results in VDAC1 oligomerization, the release of cytochrome $\mathrm{C}$ \& Smac/Diablo, and eventually apoptosis. Once present in the mitochondrial intermembrane space, $\mathrm{Ca}^{2+}$ is taken up into the mitochondrial matrix through the MCU complex, whose structure, activity, and regulation has been explained by Mammucari et al. In addition, the authors highlighted the divergent roles of $\mathrm{MCU}$ in cancer, revealing both anti- and pro-tumorigenic roles dependent on the cancer type and cancer stage. Finally, Chen and Boehning assess the role of lipidation in the regulation of different signaling pathways that impact $\mathrm{Ca}^{2+}$ homeostasis, such as Ras in apoptosis and tumor progression.

\section{MITOCHONDRIAL $\mathrm{Ca}^{2+}$ DYNAMICS IN INVASION AND METASTASIS}

Mitochondria are highly dynamic organelles undergoing constant fusion and fission governed in part by the metabolic status of the cell (19). Cancer cells, which are characterized by alterations in their metabolism, also exhibit several changes regarding mitochondrial dynamics, morphology, and cellular positioning, as discussed in the review by Pendin et al. These alterations enable cancer cells to avoid apoptosis and to cope with the sustained energy demand, particularly during migration, invasion, and metastasis. White concisely reviews the key steps controlled by $\mathrm{Ca}^{2+}$ during the invasion-metastasis cascade and reveals how changes in the $\mathrm{Ca}^{2+}$-handling components that reside in the MAMs might facilitate invasion and metastasis by impacting bioenergetics and reactive oxygen species generation. Along these lines, Hegedüs et al. provide experimental evidence that enhanced clearance of $\mathrm{Ca}^{2+}$ by increased expression of the plasma membrane $\mathrm{Ca}^{2+}$ ATPase $4 \mathrm{~b}$ induced by histone deacetylase inhibitors, and reduces migration in a melanoma cell line.

\section{PERSPECTIVES}

Cancer has a multifactorial nature. Over the last decade, it has become increasingly clear that ER-to-mitochondria communication, in particular, through the flux of $\mathrm{Ca}^{2+}$ ions, impacts a plethora of cancer hallmarks and plays a critical role in oncogenesis. The emerging insights at the molecular level in the dependence of cancer cells on ER-mitochondrial $\mathrm{Ca}^{2+}$ signaling for their survival and migration will pave the path for new therapeutic strategies and tools (20), hopefully opening up new avenues for designing anticancer treatments that exploit these dependencies and vulnerabilities.

\section{AUTHOR CONTRIBUTIONS}

CC drafted the manuscript that was reviewed and edited by PP and GB. All authors co-edited the Research Topic.

\section{ACKNOWLEDGMENTS}

CC wants to thank Alenka Lovy for her continuous support, creative power, and inspiring discussion. CC has been supported by FONDECYT grant \#1160332 (CC) and CONICYT/ FONDAP \#15150012 (CC). GB was supported by the Research 
Foundation-Flanders (FWO) through grants \#G.0634.13N, G.0C91.14N, G.0A34.16N, and G.0901.18N and by the Research Council-KU Leuven through grant \#OT/14/101. GB and PP are part of the FWO Scientific Research Community CaSign (WO.019.17N). PP is grateful to Camilla degli Scrovegni for

\section{REFERENCES}

1. Torre LA, Siegel RL, Ward EM, Jemal A, et al. Global cancer incidence and mortality rates and trends - an update. Cancer Epidemiol Biomarkers Prev (2016) 25(1):16-27. doi:10.1158/1055-9965.EPI-15-0578

2. Hanahan D, Weinberg RA. The hallmarks of cancer. Cell (2000) 100(1):57-70. doi:10.1016/S0092-8674(00)81683-9

3. Stratton MR, Campbell PJ, Futreal PA. The cancer genome. Nature (2009) 458(7239):719-24. doi:10.1038/nature07943

4. Parsons DW, Jones S, Zhang X, Lin JC, Leary RJ, Angenendt P, et al. An integrated genomic analysis of human glioblastoma multiforme. Science (2008) 321(5897):1807-12. doi:10.1126/science.1164382

5. Cairns RA, Harris IS, Mak TW. Regulation of cancer cell metabolism. Nat Rev Cancer (2011) 11(2):85-95. doi:10.1038/nrc2981

6. Hanahan D, Weinberg RA. Hallmarks of cancer: the next generation. Cell (2011) 144(5):646-74. doi:10.1016/j.cell.2011.02.013

7. Bernhard W, Rouiller C. Close topographical relationship between mitochondria and ergastoplasm of liver cells in a definite phase of cellular activity. J Biophys Biochem Cytol (1956) 2(4 Suppl):73-8. doi:10.1083/jcb.2.4.73

8. Wieckowski MR, Giorgi C, Lebiedzinska M, Duszynski J, Pinton P. Isolation of mitochondria-associated membranes and mitochondria from animal tissues and cells. Nat Protoc (2009) 4(11):1582-90. doi:10.1038/nprot. 2009.151

9. Giorgi C, Missiroli S, Patergnani S, Duszynski J, Wieckowski MR, Pinton P. Mitochondria-associated membranes: composition, molecular mechanisms, and physiopathological implications. Antioxid Redox Signal (2015) 22(12): 995-1019. doi:10.1089/ars.2014.6223

10. Bittremieux M, Parys JB, Pinton P, Bultynck G. ER functions of oncogenes and tumor suppressors: modulators of intracellular $\mathrm{Ca}(2+)$ signaling. Biochim Biophys Acta (2016) 1863(6 Pt B):1364-78. doi:10.1016/j.bbamcr. 2016.01.002

11. Kerkhofs M, Giorgi C, Marchi S, Seitaj B, Parys JB, Pinton P, et al. Alterations in $\mathrm{Ca}(2+)$ signalling via ER-mitochondria contact site remodelling in cancer. Adv Exp Med Biol (2017) 997:225-54. doi:10.1007/978-981-10-4567-7_17 continuous support. PP is supported by the Italian Ministry of Education, University and Research; the Italian Ministry of Health; Telethon (GGP15219/B); the Italian Association for Cancer Research (AIRC: IG-18624); and by local funds from the University of Ferrara.

12. Bultynck G, Parys JB. $\mathrm{Ca}(2+)$ signaling and cell death: focus on $\mathrm{Ca}(2+)$ transport systems and their implication in cell death and survival. Cell Calcium (2018) 69:1-3. doi:10.1016/j.ceca.2017.09.001

13. Parys JB, Bultynck G. $\mathrm{Ca}(2+)$ signaling and cell death: focus on the role of $\mathrm{Ca}(2+)$ signals in the regulation of cell death \& survival processes in health, disease and therapy. Cell Calcium (2017). doi:10.1016/j.ceca.2017.11.003

14. Berridge MJ, Bootman MD, Roderick HL. Calcium signalling: dynamics, homeostasis and remodelling. Nat Rev Mol Cell Biol (2003) 4(7):517-29. doi:10.1038/nrm1155

15. Marchi S, Pinton P. Alterations of calcium homeostasis in cancer cells. Curr Opin Pharmacol (2016) 29:1-6. doi:10.1016/j.coph.2016.03.002

16. Bultynck G, Campanella M. Tumor suppressive Ca2+ signaling is driven by IP3 receptor fitness. Cell Stress (2017) 1(2):73-8. doi:10.15698/cst2017.11.109

17. Lovy A, Foskett JK, Cardenas C. InsP3R, the calcium whisperer: maintaining mitochondrial function in cancer. Mol Cell Oncol (2016) 3(4):e1185563. doi:10.1080/23723556.2016.1185563

18. Glancy B, Balaban RS. Role of mitochondrial Ca2+ in the regulation of cellular energetics. Biochemistry (2012) 51(14):2959-73. doi:10.1021/bi2018909

19. Nunnari J, Suomalainen A. Mitochondria: in sickness and in health. Cell (2012) 148(6):1145-59. doi:10.1016/j.cell.2012.02.035

20. Kerkhofs M, et al. Emerging molecular mechanisms in chemotherapy: Ca2+ signaling at the mitochondria-associated endoplasmic reticulum membranes. Cell Death Dis (2018). doi:10.1038/s41419-017-0179-0

Conflict of Interest Statement: The authors declare that the research was conducted in the absence of any commercial or financial relationships that could be construed as a potential conflict of interest.

Copyright (c) 2018 Cardenas, Pinton and Bultynck. This is an open-access article distributed under the terms of the Creative Commons Attribution License (CC $B Y$ ). The use, distribution or reproduction in other forums is permitted, provided the original author(s) and the copyright owner are credited and that the original publication in this journal is cited, in accordance with accepted academic practice. No use, distribution or reproduction is permitted which does not comply with these terms 